\title{
A Novel Grammar-based Genetic Programming Approach to Clustering
}

\author{
I. De Falco, E. Tarantino \\ ICAR - CNR \\ Via P. Castellino, 111 \\ 80131 Naples, ITALY \\ ivanoe.defalco@na.icar.cnr.it \\ A. Della Cioppa \\ DIIIE - University of Salerno \\ Via Ponte don Melillo, 1 \\ 84084 Fisciano (SA), ITALY \\ adellacioppa@unisa.it
}

\author{
F. Fontanella \\ DIS - University of Naples \\ Via Claudio, 21 \\ 80125 Naples, ITALY \\ frfontan@unina.it
}

\begin{abstract}
Most of the classical methods for clustering analysis require the user setting of number of clusters. To surmount this problem, in this paper a grammar-based Genetic Programming approach to automatic data clustering is presented. An innovative clustering process is conceived strictly linked to a novel cluster representation which provides intelligible information on patterns. The efficacy of the implemented partitioning system is estimated on a medical domain by exploiting expressly defined evaluation indices. Furthermore, a comparison with other clustering tools is performed.
\end{abstract}

\section{Categories and Subject Descriptors}

H.3.3 [Information Storage and Retrieval]: Information search and retrieval-clustering, retrieval methods; I.2.8 [Artificial Intelligence]: Problem Solving, Control Methods, and Search-heuristic methods

\section{General Terms}

Algorithms, Experimentation

\section{Keywords}

Genetic programming, data clustering.

\section{INTRODUCTION}

Clustering consists in grouping a given collection of unknown patterns into meaningful clusters with no assumptions about the relationships based on some similarity criterion [8]. A variety of clustering algorithms have been developed [7]. The output of most of these algorithms is strongly dependent on user inputs of several parameters, like the number of clusters and the average dimensionality of the cluster $[18,5,4,1]$. To overcome these drawbacks, evolutionary techniques have been applied to cluster analysis $[10,6,15]$ and, later, the usage of combined strategies

Permission to make digital or hard copies of all or part of this work for personal or classroom use is granted without fee provided that copies are not made or distributed for profit or commercial advantage and that copies bear this notice and the full citation on the first page. To copy otherwise, to republish, to post on servers or to redistribute to lists, requires prior specific permission and/or a fee.

SAC'05 March 13-17, 2005, Santa Fe, New Mexico, USA

Copyright 2005 ACM 1-58113-964-0/05/0003 ...\$5.00. has been attempted $[12,2]$. As in [13], in this work the effectiveness of a a grammar-based Genetic Programming (GP) [11] framework to perform automatic data clustering is investigated. The hope is that this system, exploiting information implicitly included in the data set, allows to find an efficient partitioning with interesting correlations among patterns. To this aim a new clustering procedure, tied to an innovative way of representing clusters based on logical formulas, is proposed.

The paper is organized as follows: in Sect. 2 a formalization of data clustering is reported, while in Sect. 3 our GP-based clustering system is outlined together with its implementation details. In Sect. 4 some evaluation indices are described. The results are discussed in Sect. 5. The last section illustrates our conclusions.

\section{DATA CLUSTERING}

Each element of the database is said pattern and is represented by $\mathbf{X}=\left(x_{1}, \ldots, x_{\ell}\right)$ with $\mathbf{X} \in \mathbf{S}$, where $\mathbf{S}$ is the universe of all possible elements with $\ell$ attributes and $x_{j}$ denotes the $j$-th attribute of the pattern. A pattern set $\mathcal{X}$ with cardinality $n$ is denoted with $\mathcal{X}=\left(\mathbf{X}_{1}, \ldots, \mathbf{X}_{n}\right)$ with, in general, $\mathcal{X} \subseteq \mathbf{S}$. For numerical attributes, once defined the range for the $j$-th attribute as: $R_{j} \equiv \delta\left(\max _{i=1, \ldots, n} x_{i j}, \min _{i=1, \ldots, n} x_{i j}\right)$ where $x_{i j}$ indicates the $j$-th attribute of the $i$-th pattern, the distance between patterns chosen is the Manhattan one:

$$
d(\mathbf{X}, \mathbf{Y})=\frac{1}{\ell} \cdot \sum_{j=1}^{\ell} \frac{1}{R_{j}} \cdot\left|x_{j}-y_{j}\right|
$$

This distance is normalized with respect to the range $R_{j}$ and to the number of attributes, so as to have $0 \leq d(\mathbf{X}, \mathbf{Y}) \leq$ $1 \forall \mathbf{X}, \mathbf{Y} \in \mathcal{X}$.

Several ways are possible to represent a cluster $\mathbf{C}_{k}$. However, the most used representative is the centroid $\mathbf{P}_{k}$ defined as the average point for patterns belonging to $\mathbf{C}_{k}$. In this case, a clustering $\mathbf{C L}$ with cardinality $\mathcal{N}$ can be denoted by the list of its cluster representatives: $\mathbf{C L} \equiv\left\{\mathbf{P}_{1}, \ldots, \mathbf{P}_{\mathcal{N}}\right\}$.

To estimate the clustering quality with respect to the given data set, a scoring function $f_{s}$, expressed in terms of homogeneity $\mathcal{H}$ and separability $\mathcal{S}$ for the clustering $\mathbf{C L}$, is considered. This generic function is: $f_{s}=f(\mathcal{H}(\mathbf{C L}), \mathcal{S}(\mathbf{C L}))$ where the dependence on $\mathcal{X}$ is implicit in the computation of $\mathcal{H}(\mathbf{C L})$ and $\mathcal{S}(\mathbf{C L})$. Denoting with $w_{k}$ the cardinality for a cluster $\mathbf{C}_{k}$, homogeneity is defined as:

$$
\mathcal{H}\left(\mathbf{C}_{k}\right) \equiv-\frac{\sum_{\mathbf{X} \in \mathbf{C}_{k}}\left[d\left(\mathbf{X}, \mathbf{P}_{k}\right)\right]}{w_{k}}
$$


Hence we can define $\mathcal{H}(\mathbf{C L})$ as weighted average of homogeneity of clusters, and $\mathcal{S}(\mathbf{C L})$ as the weighted average of distances among clusters. Formally, we have:

$$
\begin{gathered}
\mathcal{H}(\mathbf{C L}) \equiv \frac{\sum_{i=1}^{m} w_{i} \cdot \mathcal{H}\left(\mathbf{C}_{i}\right)}{\sum_{i=1}^{m} w_{i}} \\
\mathcal{S}(\mathbf{C L}) \equiv \frac{\sum_{i=1}^{m-1} \sum_{j=i+1}^{m} w_{i} \cdot w_{j} \cdot d\left(\mathbf{C}_{i}, \mathbf{C}_{j}\right)}{\sum_{i=1}^{m-1} \sum_{j=i+1}^{m} w_{i} \cdot w_{j}}
\end{gathered}
$$

Distance between clusters is computed as the distance between respective centroids by using (1).

\section{OUR GP SYSTEM FOR CLUSTERING}

\subsection{Clustering Encoding}

In our system, a cluster prototype is a logical formula $E$ composed by a variable number of predicates and a clustering is represented by a variable number of such formulas. Any GP individual encodes a clustering as a tree structure. A formula generator, based on the context-free grammar in Table 1, provides the starting population. This grammar ensures the syntactic correctness of the formulas. The tree nodes can be either terminal or nonterminal symbols. These latter are indicated in capital letter. Moreover, the terminal symbol $\$$ is introduced as formula delimiter. Since the grammar is non-deterministic, the action carried out by a rule is chosen based on the fixed values of probabilities shown in the table so as to reduce the probability of generating too long formulas. However, in addition an upper limit has been imposed to the total number of predicates contained in the set of formulas representing a clustering and individuals which overcome it will be generated anew. The generator starts by applying the first rule and then the remaining ones as long as they are called upon. The aim is to create a set of logical formulas different in terms of size, shape and functionality. It can be noted that the grammar allows to generate individuals composed by at least two formulas. For the predicates of each formula $E_{k}$ conditions on some of the attributes $a_{i}$ of the database patterns are set. When all the predicates of this formula are satisfied by the attribute values of the pattern, the formula is said to match the pattern itself. The matching between a generic pattern and the formulas is effected by an interpreter. All the formulas of the individual will be used to perform the clustering process which takes place in two steps:

1. assign all the patterns which are matched by the formulas (the formulas which match no pattern are not considered in the step 2 and they simply represent introns in the genotype):

- a pattern is matched by one formula only: it is assigned to the related cluster;

- a pattern is matched by more than one formula with different number of predicates: the pattern is assigned to the formula with the greatest number of matched predicates ${ }^{1}$;

- a pattern is satisfied by more than one formula with the same number of predicates: the pattern

\footnotetext{
${ }^{1}$ The aim is to assign the pattern to the formula which delimits a smaller part of feature space, so to obtain a more specialized cluster.
}

Table 1: The grammar for the random program generator.

\begin{tabular}{clr} 
Rule number & Rule & Probability \\
\hline 1 & $S \longrightarrow(Q) B A \$(Q) B A E$ & 1.0 \\
2 & $Q \longrightarrow L \mid M$ & equiprobable \\
3 & $L \longrightarrow \Lambda \mid C$ & $0.7,0.3$ \\
4 & $M \longrightarrow C \vee M \mid C$ & $0.7,0.3$ \\
5 & $B \longrightarrow \vee \mid \wedge$ & equiprobable \\
6 & $A \longrightarrow(Q) B A \mid(Q)$ & $0.3,0.7$ \\
7 & $E \longrightarrow \$(Q) B A E \mid \$$ & $0.6,0.4$ \\
8 & $C \longrightarrow(P N Z)$ & 1.0 \\
9 & $P \longrightarrow a_{0}\left|a_{1}\right| \ldots \mid a_{32}$ & equiprobable \\
10 & $Z \longrightarrow 0|1| 2 \mid 3$ & equiprobable \\
11 & $N \longrightarrow \geq|\leq|=|>|<$ & equiprobable
\end{tabular}

is classified as undefined and it is not assigned at this stage.

2. assign the patterns which are not matched by any formula of the clustering and the undefined ones:

- for each formula, compute the average points of all the patterns assigned so far to it: this point is called matching centroid;

- assign any pattern to the cluster whose matching centroid is the closest.

This process, hereinafter referred to as clustering, permits to combine the advantages of a clustering whose representation is based on logical formulas with those of a classical representation based on centroids. In fact, the former provides a feature extraction and correlation capability and the latter makes the process able to perform a complete clustering in which all the patterns are assigned. Hence, the phenotype $\mathbf{F}$, representing the clustering, is constituted by a variable number of formulas, each of which is representative of a cluster. Specifically, $\mathbf{F} \equiv\left\{E_{1}, E_{2}, \ldots, E_{\mathcal{N}}\right\}$ where $\mathcal{N}$ is the number of formulas.

\subsection{Fitness Function}

After performing the clustering process, an appropriate fitness function must be chosen to evaluate the quality of the segmentation based on $\mathbf{F}$. A penalty term linked to the number of clusters could be added in the fitness, with the aim to avoid a probable overfitting of the solution with respect to the given database due to uncontrolled increase in the number of clusters. Nevertheless, this would mean to establish a limit to this number, by using some a priori knowledge on the application domain, which would make our algorithm dependent on user skill. To surmount this problem, following [17], we consider as fitness function a linear combination of intra-cluster homogeneity and intercluster separability:

$$
f(\mathbf{F})=\mathcal{H}(\mathbf{F})+\mu \cdot \mathcal{S}(\mathbf{F})
$$

where $\mu$ is a scaling factor. Since $d(\mathbf{X}, \mathbf{Y}) \leq 1$, we have that $-1 \leq \mathcal{H}(\mathbf{F}) \leq 0$ and $0 \leq \mathcal{S}(\mathbf{F}) \leq 1$, and, therefore, $-1 \leq f(\overline{\mathbf{F}}) \leq \mu$. In this way, data clustering becomes a problem of direct maximization for homogeneity and separability independently of number of clusters $\mathcal{N}$, and depending on a scale factor liable for balance between them. Thus the fitness $f(\mathbf{F})$ does not explicitly depend on $\mathcal{N}$, yet as the 
parameter $\mu$ varies a control on the number of clusters will be indirectly achieved. In fact the relative weights for $\mathcal{H}$ and $\mathcal{S}$ change as $\mu$ varies. Namely it is to note that, for low values of $\mu, \mathcal{H}$ is dominant in (4) and as $\mathcal{H}$ increases $\mathcal{N}$ tends to grow, while, when $\mu$ grows, the value of $\mathcal{S}$ assumes a more significant weight in (4) and, consequently, its increment tends to let $\mathcal{N}$ decrease. To calculate more accurately $\mathcal{H}(\mathbf{F})$ and $\mathcal{S}(\mathbf{F})$, the centroids of all the actual clusters achieved at the end of the clustering process are evaluated and their values are used in (2) and in (3).

\subsection{Genetic Operators}

The phenotypes are encoded as derivation trees, generated by the grammar, representing the genotypes the genetic operators work with. The new elements are generated by means of two operators, crossover and mutation, which preserve the syntactic correctness of the formulas.

The crossover operates by randomly selecting the same nonterminal node in the two individuals to be crossed and then swapping the corresponding derivation subtrees.

Differently from classical GP, the mutation works on any obtained offspring by randomly choosing a nonterminal node in the individual to be mutated, and then the corresponding production rule is activated in order to generate a new subtree or to substitute a leaf node.

\section{EVALUATION INDICES}

Evaluation indices can be defined and exploited to quantitatively estimate a posteriori the degree of usefulness and meaningfulness of found clusters. In general, given a clustering and a data set where classes are known, a table between the $p$ classes and the $s$ found clusters can be conceived. This table corresponds to a matrix $\mathbf{B}$ where each element $b_{i j}$ represents the number of patterns in the data set belonging to class $i$ assigned to cluster $j$. Two indicators can be defined: the Class Addressing Index $\mathcal{A}$ and the Class Gathering In$\operatorname{dex} \mathcal{G}$. For the $j$-th cluster:

$$
\mathcal{A}_{j}=\sum_{i=1}^{p} \frac{b_{i j}^{2}}{\left(\sum_{i=1}^{p} b_{i j}\right)^{2}}
$$

This index represents a normalized weighted average and it measures the ability of a cluster to address towards a single class. Its value is 1 in the best case, in which the cluster represents only one class, and it decreases as the number of the addressed classes increases. In the worst case its value is equal to $\frac{1}{p}$. We define $\mathcal{A}$ for the whole clustering as the weighted average for indices of each cluster:

$$
\mathcal{A}=\frac{\sum_{j=1}^{s} w_{j} \cdot \mathcal{A}_{j}}{\sum_{j=1}^{s} w_{j}}
$$

where $w_{j}$ denotes the weight of $j$-th cluster, i.e. the number of patterns assigned to $j$-th cluster. Its variation range is the same of $\mathcal{A}_{j}$. In a similar way, for the $i$-th class:

$$
\mathcal{G}_{i}=\sum_{j=1}^{s} \frac{b_{i j}^{2}}{\left(\sum_{j=1}^{s} b_{i j}\right)^{2}}
$$

It indicates as a single class is subdivided among the clusters. Also in this case, its value is 1 when all the elements of one class are grouped in one cluster, while it decreases down to $\frac{1}{s}$ as the number of clusters in which a class is subdivided increases. For the whole clustering

$$
\mathcal{G}=\frac{\sum_{i=1}^{p} w_{i} \cdot \mathcal{G}_{i}}{\sum_{i=1}^{p} w_{i}}
$$

where $w_{i}$ denotes the weight of $i$-th class, i.e. the number of patterns belonging to $i$-th class. The variation range is the same as that of $\mathcal{G}_{i}$.

For a global evaluation we take into account the so-called Correspondence Index $\mathcal{I}_{c}=\mathcal{A} \cdot \mathcal{G}$. This index permits to estimate the utility of detected clusters in addressing towards the classes. Its range is $(1 / p) \cdot(1 / s) \leq \mathcal{I}_{c} \leq 1.0$.

\section{EXPERIMENTAL RESULTS}

A database, constituted by 366 clinical dermatological cases, available at UCI site [14] has been considered. Each instance has 34 attributes in the range $[0,3]$ where 0 indicates the absence of the attribute and 3 the presence at its maximum degree, while 1 and 2 denote intermediate values. Such a database is already subdivided into 6 classes on the base of medical belief.

The basic evolutionary parameters have been set by preliminary trials. In particular, population size $p_{s}=100$, mutation probability $p_{m}=0.9$, crossover probability $p_{c}=0.9$ and number of generations $n_{g}=250$. The selection has been the tournament with size $\mathcal{T}=10$. Besides the maximum number of predicates in the set of formulas has been fixed equal to 200.

The experiments aim at studying the variation of $\mathcal{H}$ and $\mathcal{S}$ as a function of the scale factor $\mu$ in the range $[0.0,1.0]$ with step 0.1. In Table 2 the average values of $\mathcal{H}, \mathcal{S}$ and $\mathcal{N}$ for each value of $\mu$ are reported. These values have been obtained performing only 10 runs for each value of the scale factor considering the low standard deviations attained. The objective is to find the best $\mu$ in terms of $\mathcal{H}$ and $\mathcal{S}$. In order to individuate it, we have defined a hypothetical 'optimal point' (shown in bold in table) obtained considering the best of $\mathcal{H}$ and $\mathcal{S}$ among those found during our trials. Among all the different values of $\mathcal{H}$ and $\mathcal{S}$ in table, the closest to this 'optimal point', in terms of distance, has resulted to be that with $\mu=0.7$. Table 2 shows also that as $\mu$ increases, the average number of clusters decreases which reveals that an implicit control on $\mathcal{N}$ can be actually achieved by suitably setting values for the scaling factor.

By using $\mu=0.7$, the behavior during the best run in terms of fitness is shown in Fig. 1. In particular, on the left the average fitness of the population and the fitness of the best individual are reported as a function of the generation number. As it can be noted, there is a startup phase for the best fitness in which its value is almost constant and much higher than the average one. Such a behavior is due to the predominance of $\mathcal{S}(\mathbf{F})$ in the fitness function which keeps low the related best number of clusters as it can be seen in Fig. 1 (right). Starting from about 40 generations this fitness increases constantly until around generation 100 . This is owed to the improvements of $\mathcal{H}(\mathbf{F})$ as confirmed by the increment in the corresponding number of clusters with respect to the initial phase. Then the process continues with a lower increase until about 150 generations. During this phase the number of clusters remains constant. The last phase, up to generation 250, shows no significant variation in terms of the best fitness and the decrease and the stabilization in the associated number of clusters. 
Table 2: Average values of homogeneity and separability as a function of $\mu$

\begin{tabular}{cccccccccccc}
$\mu$ & 0.0 & 0.1 & 0.2 & 0.3 & 0.4 & 0.5 & 0.6 & 0.7 & 0.8 & 0.9 & 1.0 \\
\hline $\mathcal{H}$ & -0.106 & $\mathbf{- 0 . 1 0 5}$ & -0.111 & -0.115 & -0.119 & -0.120 & -0.119 & -0.125 & -0.136 & -0.140 & -0.133 \\
$\mathcal{S}$ & 0.234 & 0.237 & 0.244 & 0.248 & 0.253 & 0.254 & 0.252 & 0.259 & 0.265 & $\mathbf{0 . 2 7 4}$ & 0.265 \\
$\mathcal{N}$ & 15.6 & 14.6 & 12.2 & 9.8 & 7.5 & 6.7 & 4.9 & 4.5 & 4.3 & 4.2 & 3.4
\end{tabular}
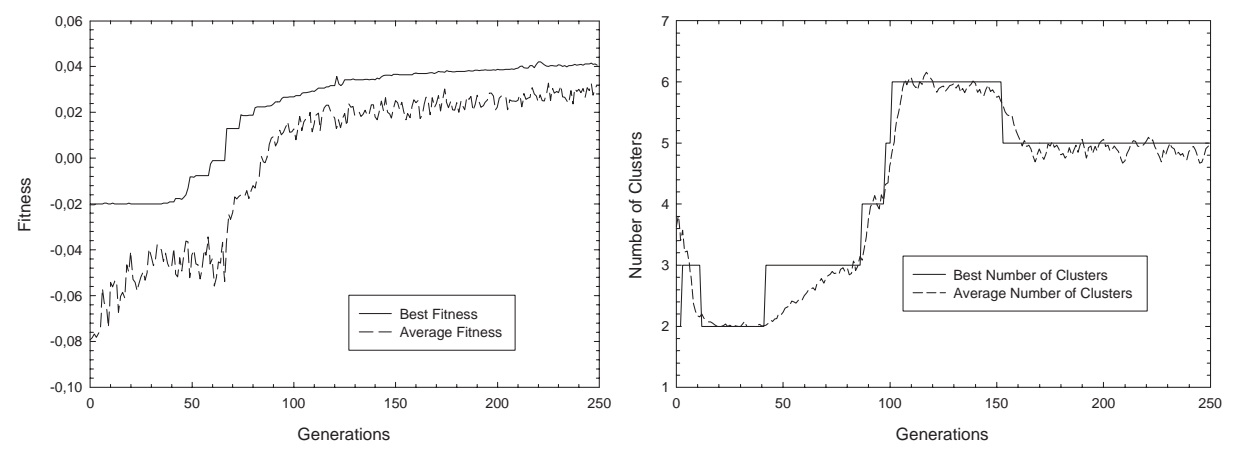

Figure 1: Behavior of fitness (left) and number of clusters (right) during the best run.

Table 3: Classes-clusters $(p \backslash s)$ relationship for the solution with $\mu=0.7$

\begin{tabular}{c|ccccc}
$p \backslash s$ & 1 & 2 & 3 & 4 & 5 \\
\hline 1 & 0 & 112 & 0 & 0 & 0 \\
2 & 0 & 0 & 0 & 0 & 61 \\
3 & 0 & 0 & 0 & 72 & 0 \\
4 & 0 & 0 & 0 & 0 & 49 \\
5 & 50 & 0 & 0 & 0 & 2 \\
6 & 0 & 0 & 19 & 0 & 1
\end{tabular}

In Table 3 the clustering obtained during the best run above is reported. For the medical database considered, it should be observed that the second and the fourth cluster address perfectly the first and the third class respectively. Almost the same holds for the first and the third cluster. Moreover it is clear that the second and the fourth classes have not been distinguished. Nonetheless, this result was expected since it is known that these classes are hard to separate by medical experts as well. This fact yields that the value of $\mathcal{A}=0.8367$ is satisfactory but not so close to the maximum. Furthermore, it is worth observing that each class has been almost optimally gathered in one cluster. This is confirmed by the very good value of $\mathcal{G}=0.989$. Thus the global index $\mathcal{I}_{c}$ is equal to 0.8275 . These values assure that the clustering achieved is effective.

As an example of the clustering, we report in the following the formula representative of the first cluster in Table 3:

Cluster 1: $\left(\left(a_{17}=2\right) \vee\left(a_{10}=1\right) \vee\left(a_{15}>0\right)\right) \wedge\left(\left(a_{27}=\right.\right.$ 1) $\left.\vee\left(a_{6}>1\right)\right) \wedge\left(\left(a_{16}=2\right) \vee\left(a_{2}=2\right) \vee\left(a_{30}=3\right) \vee\left(a_{19}=\right.\right.$ 1) $\left.\vee\left(a_{3}=2\right) \vee\left(a_{15}<2\right)\right) \wedge\left(a_{14} \geq 2\right)$

As it is evident this novel representation has the advantage of providing explicit information both on the features of the patterns satisfying each formula and on their values.

In Table 4 the average values of all the indices, related to the best solutions in terms of fitness during 10 runs, are shown as a function of $\mu$. It should be noted that the value $\mathcal{I}_{c}$ obtained for $\mu=0.7$ is always the best. This seems to validate a posteriori its choice.

\subsection{Comparison findings}

The approach proposed has been compared with other two classical clustering algorithms, the k-means [9] and the Expectation-Maximization (EM) [3]. These tools are part of Weka, a collection of algorithms for data mining [16]. The comparison has been conducted on the basis of the defined evaluation indices.

The k-means method requires a preliminary setting of the desired number of clusters into which the database has to be partitioned. Ten runs have been performed with different initial seeds for any value of the number of clusters in the range $[2-10]$. In Table 5 the average values of all the indices are reported as a function of the imposed value of number of clusters $\mathcal{N}$. From this table it can be observed that, as expected, the average value of $\mathcal{A}$ grows as $\mathcal{N}$ increases, while the average value of $\mathcal{G}$ decreases as $\mathcal{N}$ grows. Note that the best average value for $\mathcal{I}_{c}$ is obtained for $\mathcal{N}=5$ and it is lower than that carried out by our system. As regards the best results, $\mathcal{A}$ values range within 0.461 and 0.946 , while for low imposed number of clusters (from 2 up to 5 ) the best values for $\mathcal{G}$ are almost constant ranging within 1 and 0.989. Starting from 6 clusters on these values of $\mathcal{G}$ decrease from 0.973 down to 0.664 . The related best values for $\mathcal{I}_{c}$ are within 0.461 and 0.852 . It is to underline that also this last value has been found for $\mathcal{N}=5$. Moreover, though it is better than that found by means of our approach, it should be perceived that this result has been achieved providing the system with a priori knowledge by imposing the number of clusters. However, this comparison confirms the goodness of our approach in facing this clustering problem even if the running times are substantially higher.

The choice of EM as further comparison algorithm is due to the fact that, like our system, it does not require a priori information about the number of clusters. We have used the default parameter setting as reported in Weka, according to which the maximum number of iterations is 100 , and the minimum standard deviation for each attribute in each cluster is $1.0 \cdot 10^{-6}$. We have carried out 10 runs with different initial seeds finding solutions differing in the number of clusters and in the assignment of database instances to 
Table 4: Average index values as a function of $\mu$

\begin{tabular}{ccccccccccccc}
$\mu$ & 0.0 & 0.1 & 0.2 & 0.3 & 0.4 & 0.5 & 0.6 & 0.7 & 0.8 & 0.9 & 1.0 \\
\hline $\mathcal{A}$ & 0.5945 & 0.925 & 0.803 & 0.927 & 0.746 & 0.8154 & 0.8274 & 0.8167 & 0.665 & 0.6642 & 0.615 \\
$\mathcal{G}$ & 0.835 & 0.735 & 0.783 & 0.816 & 0.704 & 0.938 & 0.952 & 0.979 & 0.949 & 0.916 & 0.933 \\
$\mathcal{I}_{c}$ & 0.4964 & 0.6799 & 0.6287 & 0.7564 & 0.5252 & 0.7648 & 0.7877 & 0.7995 & 0.6311 & 0.6084 & 0.5738
\end{tabular}

Table 5: Average index values as a function of the imposed number of clusters $\mathcal{N}$

\begin{tabular}{cccccccccc}
$\mathcal{N}$ & 2 & 3 & 4 & 5 & 6 & 7 & 8 & 9 & 10 \\
\hline $\mathcal{A}$ & 0.447 & 0.608 & 0.738 & 0.806 & 0.809 & 0.856 & 0.878 & 0.887 & 0.9 \\
$\mathcal{G}$ & 0.998 & 0.980 & 0.958 & 0.919 & 0.844 & 0.798 & 0.714 & 0.662 & 0.611 \\
$\mathcal{I}_{c}$ & 0.446 & 0.596 & 0.707 & 0.741 & 0.683 & 0.683 & 0.627 & 0.587 & 0.550
\end{tabular}

clusters. The number of clusters present in all the suggested solutions varies within 5 and 8 . The comparison with our tool is still effected in terms of the evaluation indices. The average values for $\mathcal{A}$ and $\mathcal{G}$ have resulted to be 0.714 and 0.864 respectively. The average value for $\mathcal{I}_{c}$ has turned out to be 0.618 , lower than that shown by our tool which is 0.7995 . The best values are $\mathcal{A}=0.788, \mathcal{G}=0.916$ and $\mathcal{I}_{c}=0.721$. The proposed number of clusters for this solution is equal to 6 . Therefore, this comparison confirms that our approach, despite its higher execution times, is effective also with respect to the EM algorithm.

\section{CONCLUSIONS}

In this paper, the flexibility of a GP framework in performing automatic clustering has been investigated. The implemented system is capable of detecting clusters orienting towards classes. As a data clustering algorithm, satisfactory results have been achieved. Furthermore, thanks to GP, an innovative mode of representing clusters, based on logical formulas, has been introduced. Not only has this allowed to perform an original clustering process which exploits the advantages of both a new representation and a classical one, it has also permitted the discovery of common relationships of patterns belonging to a same cluster. Comparison with other traditional clustering tools in terms of evaluation indices has demonstrated the effectiveness of our system.

\section{REFERENCES}

[1] Aggarwal, C. and Yu, P. S. Finding generalized projected clusters in high dimensional spaces. In Proc. ACM SIGMOD Int. Conf. on Management of Data, 2000, 70-81.

[2] Babu, G. P. and Marty, M. N. Clustering with evolutionary strategies. Pattern Recognition, 27,2 (1994), 321-329.

[3] Dempster, A. P., Laird, N. M., and Rubin, D. B. Maximum Likelihood from Incomplete Data via the EM algorithm. Journal of the Royal Statistical Society, Series B., 39, 1 (1977), 1-38.

[4] Fraley, C. and Raftery, A. How many clusters? Which clustering method? Answers via model-based cluster analysis. The Computer Journal, 41, 8 (1998), $578-588$.

[5] Guha, S., Rastogi, R., and Shim, K. CURE: an efficient clustering algorithm for large databases. In
Proc. ACM SIGMOD Int. Conf. on Management of Data, 1998, 73-84.

[6] Hall, L. O., Ozyurt, B., and Bezdek, J. C. Clustering with a genetically optimized approach. IEEE Trans. on Evolutionary Computation, 3, 2 (1999), 103-112.

[7] Han, J. and Kamber, M. Data mining: concepts and techniques. Morgan Kaufmann, 2001.

[8] Hand, D. J., Mannila, H., and Smyth, P. Principles of data mining. MIT Press, 2001.

[9] Hartigan, J. Clustering algorithms. Wiley \& Sons, New York, 1975.

[10] Jain, A. K., Murty, M. N., and Flynn, P. J. Data clustering: a review. ACM Computing Surveys, 31,3 (1999), 264-323.

[11] Koza, J. R. Genetic Programming: on programming computers by means of natural selection and genetics. The MIT Press, Cambridge, MA, 1992.

[12] Lee, C. Y. and Antonsson, E. K. Dynamic partitional clustering using evolutionary strategies. In Proc. of the 3rd Asia-Pacific Conference on Simulated Evolution and Learning. IEEE Press, Nagoya, Japan, 2000.

[13] Leung, K. S. and Wong, M. L. Data mining using grammar based Genetic Programming and applications. Kluwer, 2000.

[14] Murphy, P. M. and Aha, D. W. UCI Repository of machine learning databases. University of California, Dept. of Information and Computer Science. Available at www.ics.uci.edu/ mlearn/MLRepository.html

[15] Sarafis, I., Zalzala, A. M. S., and Trinder, P. W. A genetic rule-based data clustering toolkit. In Proc. of the IEEE Congress on Evolutionary Computation, 2002, 1238-1243.

[16] Weka3.4: Data mining software in Java. Available at www.cs.waikato.ac.nz/ ml/weka/

[17] Yip, A. M. A scale dependent data clustering model by direct maximization of homogeneity and separation. In Proc. Mathematical challenges in scientific data mining IPAM. 14-18 January 2002.

[18] Zhang, T., Ramakrishnan, R., and Livny, M. BIRCH: an efficient data clustering method for very large databases. In Proc. ACM SIGMOD Int. Conf. on Management of Data, 1996, 103-114. 